\title{
Invariants of linear parabolic differential equations
}

\author{
N.H. Ibragimov ${ }^{a}$, S.V. Meleshko ${ }^{\text {b,* }}$, E. Thailert ${ }^{\text {b }}$ \\ ${ }^{a}$ Research Centre ALGA: Advances in Lie Group Analysis, Department of Mathematics and Science, \\ Blekinge Institute of Technology, SE-371 79 Karlskrona, Sweden \\ ${ }^{\mathrm{b}}$ School of Mathematics, Institute of Science, Suranaree University of Technology, 111 Unuversity Avenue, \\ Nakhon Ratchasima 30000, Thailand
}

Received 18 February 2006; received in revised form 31 March 2006; accepted 31 March 2006

\section{Abstract}

The paper is dedicated to construction of invariants for the parabolic equation

$$
u_{t}+a(t, x) u_{x x}+b(t, x) u_{x}+c(t, x) u=0 .
$$

We consider the equivalence group given by point transformations and find all invariants up to seventh-order, i.e. the invariants involving the derivatives up to seventh-order of the coefficients $a, b$ and $c$ with respect to the independent variables $t, x$.

(c) 2006 Elsevier B.V. All rights reserved.

PACS: $02.30 . \mathrm{Jr} ; 02.20 . \mathrm{Tw}$

Keywords: Parabolic equations; Equivalence transformation; Semi-invariants; Invariants

\section{Introduction}

We consider the standard linear second-order parabolic partial differential equations in two independent variables:

$$
u_{t}+a(t, x) u_{x x}+b(t, x) u_{x}+c(t, x) u=0, \quad a(t, x) \neq 0
$$

Recall that the well-known group of equivalence transformations for Eq. (1) (given in [1]), i.e. the changes of variables $t, x$ and $u$ that do not change the form of Eq. (1), is composed of the linear transformation of the dependent variable,

$$
\bar{u}=\sigma(t, x) u,
$$

\footnotetext{
${ }^{*}$ Corresponding author. Tel.: +66 44224382; fax: +66 224185.

E-mail address: sergey@math.sut.ac.th (S.V. Meleshko).
} 
and the following change of the independent variables:

$$
\bar{t}=\phi(t), \quad \bar{x}=\psi(t, x),
$$

where $\sigma(t, x), \phi(t)$ and $\psi(t, x)$ are arbitrary functions obeying the invertibility conditions, $\sigma(t, x) \neq 0, \phi^{\prime}(t) \neq 0$ and $\psi_{x}(t, x) \neq 0$. The form invariance of Eq. (1) means that the transformations (2), (3) map Eq. (1) into an equation of the same form:

$$
\bar{u}_{\bar{t}}+\bar{a}(\bar{t}, \bar{x}) \bar{u}_{\bar{x} \bar{x}}+\bar{b}(\bar{t}, \bar{x}) \bar{u}_{\bar{x}}+\bar{c}(\bar{t}, \bar{x}) \bar{u}=0 .
$$

Eqs. (1) and (1') connected by an equivalence transformation are called equivalent equations.

An invariant of Eq. (1) is a function

$$
J\left(a, b, c, a_{t}, a_{x}, b_{t}, b_{x}, c_{t}, c_{x}, a_{t t}, a_{t x}, a_{x x}, \ldots, c_{x x}, \ldots\right)
$$

that remains unaltered under the equivalence transformations (2) and (3). It means that $J$ has the same value for equivalent Eqs. (1) and $\left(1^{\prime}\right)$ :

$$
J\left(a, b, c, a_{t}, \ldots, c_{x x}, \ldots\right)=J\left(\bar{a}, \bar{b}, \bar{c}, \bar{a}_{\bar{t}}, \ldots, \bar{c}_{\bar{x} \bar{x}}, \ldots\right) .
$$

If $J$ is invariant only under the transformation (2) it is termed a semi-invariant [2]. The order of an invariant (or semi-invariant) $J$ is identified with the highest order of derivatives of $a, b, c$ involved in $J$.

Semi-invariants of hyperbolic equations (termed the Laplace invariants) have been known since the 1770s. Recently there have been considerable interest in invariants of parabolic equations. The first step toward solving the problem of invariants for parabolic equations was made in [2] where the semi-invariant of the secondorder

$$
K=2 c_{x} a^{2}-b_{t} a-b_{x x} a^{2}-b_{x} b a+b_{x} a_{x} a+\frac{1}{2} b^{2} a_{x}+b a_{t}+b a_{x x} a-b a_{x}^{2}
$$

was found. It was also shown there that $K$ and the coefficient $a(t, x)$ provide a basis of semi-invariants. This solves the problem of semi-invariants. Namely, any semi-invariant $J$ of an arbitrary order involves only $a$ and $K$ together with their derivatives of an appropriate order, i.e.

$$
J=J\left(a, a_{t}, a_{x}, a_{t t}, a_{t x}, a_{x x}, \ldots, K, K_{t}, K_{x}, K_{t t}, K_{t x}, K_{x x}, \ldots\right) .
$$

Furthermore, it follows from this result that the invariants of Eq. (1) with respect to the general equivalence group can be obtained by subjecting the functions (5) to the condition of invariance under the change (3) of the independent variables.

The method and result of [2] were used in [3] for investigating invariants and invariant equations up to fifthorder with respect to the joint transformations (2) and (3). It has been shown in [3] that Eq. (1) has no invariants up to fifth-order and that it has precisely one invariant equation of the fifth-order, namely the equation

$$
\lambda=0 .
$$

The quantity $\lambda$ is defined by

$$
\begin{aligned}
\lambda= & 4 a\left(2 a K_{x x}-5 a_{x} K_{x}\right)-12 K\left(a a_{x x}-2 a_{x}^{2}\right)+a_{x}\left(4 a a_{t t}-9 a_{x}^{4}\right)-12 a_{t} a_{x}\left(a_{t}+2 a_{x}^{2}\right) \\
& +4 a\left(3 a_{t}+6 a_{x}^{2}-5 a a_{x x}\right) a_{t x}+2 a a_{x}\left(16 a_{t} a_{x x}-12 a a_{x x}^{2}+15 a_{x}^{2} a_{x x}\right)-4 a^{2} a_{t t x}-12 a^{2} a_{x} a_{t x x} \\
& -4 a^{2} a_{x x x}\left(2 a_{t}-4 a a_{x x}+3 a_{x}^{2}\right)+8 a^{3} a_{t x x x}-4 a^{4} a_{x x x x x} .
\end{aligned}
$$

and is termed a relative invariant due to the invariance of Eq. (6) with respect to the equivalence transformations (2) and (3). It is demonstrated in [3] that Eq. (6) provides a necessary and sufficient condition for Eq. (1) to be equivalent to the heat equation.

In the present paper, we find all invariants and invariant equations of the sixth- and seventh-orders. Since $\lambda=0$ singles out the heat equation and all equations equivalent to the heat equation, we exclude these equations and assume in what follows that $\lambda=0$. Under this assumption, we prove the following result. 
Theorem. An arbitrary Eq. (1) with $\lambda=0$ has one invariant of the sixth-order:

$$
\Lambda_{1}=\frac{2 a \lambda_{x}-5 \lambda a_{x}}{\lambda^{6 / 5}}
$$

and one invariant of the seventh-order:

$$
\Lambda_{2}=\frac{2 a^{2} \lambda_{x x}-9 a a_{x} \lambda_{x}+5\left(3 a_{x}^{2}-a a_{x x}\right) \lambda}{\lambda^{7 / 5}} .
$$

Furthermore, there are additional invariants of the seventh-order in the following particular cases.

(A) The family of Eq. (1) obeying the invariant conditions

$$
5 \Lambda_{2}-3 \Lambda_{1}^{2}=0, \quad \Lambda_{1} \neq 0
$$

has the invariant

$$
\Lambda_{3}=\frac{a}{\lambda^{8 / 5}}\left[a_{x} \lambda_{t}+2 a_{t} \lambda_{x}-\frac{12}{5 \lambda} a \lambda_{t} \lambda_{x}+2 a \lambda_{t x}-5 \lambda a_{t x}\right] .
$$

(B) The family of Eq. (1) defined by two invariant equations

$$
\Lambda_{1}=0, \quad \Lambda_{2}=0
$$

has the invariant

$$
\begin{aligned}
\Lambda_{4}= & \frac{1}{4 \lambda^{9 / 5}}\left[10 \lambda a^{2}\left(3 a_{x} a_{x x x}-2 a a_{x x x x}+3 a_{x x}^{2}-4 a_{t x x}\right)+5 \lambda a\left(8 a_{t} a_{x x}-8 a_{t t}+16 a_{x} a_{t x}-15 a_{x}^{2} a_{x x}-8 K_{x}\right)\right. \\
& +2 \lambda\left(50 a_{t}^{2}-4 a_{t} a_{x}^{2}+15 a_{x}^{4}+40 a_{x} K\right)+a \lambda_{x}\left(8 a_{x} a_{t}+6 a a_{x} a_{x x}-4 a^{2} a_{x x x}-8 a a_{t x}-3 a_{x}^{3}-8 K\right) \\
& \left.-40 a a_{t} \lambda_{t}+8 a^{2} \lambda_{t t}\right]-\frac{3}{5 \lambda^{14 / 5}}\left(2 a \lambda_{t}-5 \lambda a_{t}\right)^{2} .
\end{aligned}
$$

(C) The family of Eq. (1) obeying the invariant conditions

$$
\Lambda_{1}=0, \quad \Lambda_{2} \neq 0
$$

has the invariant

$$
\Lambda_{5}=4 \Lambda_{2} \Lambda_{4}-3 \Lambda_{3}^{2} .
$$

\section{Generalities}

\subsection{Equivalence group}

For obtaining invariants we use the Lie approach. This approach consists of finding an equivalence group of point transformations, and finding its invariants by solving a system of homogeneous linear equations. Let us recall the method for obtaining an equivalence group. Consider a parabolic equation (1). Since the functions $a$, $b, c$ depend on the independent variables $t, x$ only, the equivalence group should leave invariant the equations

$$
a_{u}=0, \quad b_{u}=0, \quad c_{u}=0 .
$$

Let the generator of a one-parameter equivalence group be

$$
X^{e}=\xi^{t} \frac{\partial}{\partial t}+\xi^{x} \frac{\partial}{\partial x}+\zeta^{u} \frac{\partial}{\partial u}+\zeta^{a} \frac{\partial}{\partial a}+\zeta^{b} \frac{\partial}{\partial b}+\zeta^{c} \frac{\partial}{\partial c}
$$

where the coefficients $\xi^{t}, \ldots, \zeta^{c}$ may depend, in general, depend on the variables $t, x, u, a, b, c$. The coefficients of the prolonged operator

$$
\widetilde{X}^{e}=X^{e}+\zeta^{u_{t}} \frac{\partial}{\partial u_{t}}+\zeta^{u_{x}} \frac{\partial}{\partial u_{x}}+\zeta^{u_{x x}} \frac{\partial}{\partial u_{x x}}+\zeta^{a_{u}} \frac{\partial}{\partial a_{u}}+\zeta^{b_{u}} \frac{\partial}{\partial b_{u}}+\zeta^{c_{u}} \frac{\partial}{\partial c_{u}}
$$


are defined by the prolongation formulae

$$
\begin{array}{lc}
\zeta^{u_{t}}=D_{t}^{e} \zeta^{u}-u_{t} D_{t}^{e} \xi^{t}-u_{x} D_{t}^{e} \xi^{x}, & \zeta^{u_{x}}=D_{x}^{e} \zeta^{u}-u_{t} D_{x}^{e} \xi^{t}-u_{x} D_{x}^{e} \xi^{x}, \\
\zeta^{u_{x x}}=D_{x}^{e} \xi^{u_{x}}-u_{x t} D_{x}^{e} \xi^{t}-u_{x x} D_{x}^{e} \xi^{x}, & \zeta^{a_{u}}=D_{u} \zeta^{a}-a_{t} D_{u} \xi^{t}-a_{x} D_{u} \xi^{x}, \\
\zeta^{b_{u}}=D_{u} \zeta^{b}-b_{t} D_{u} \xi^{t}-b_{x} D_{u} \xi^{x}, & \zeta^{c_{u}}=D_{u} \zeta^{b}-c_{t} D_{u} \xi^{t}-c_{x} D_{u} \xi^{x} .
\end{array}
$$

Here the operators $D_{t}^{e}, D_{x}^{e}$ are operators of the total derivatives with respect to $t$ and $x$, respectively, where the space of the independent variables consists of $t$ and $x$,

$$
\begin{aligned}
& D_{t}^{e}=\frac{\partial}{\partial t}+u_{t} \frac{\partial}{\partial u}+\left(a_{t}+u_{t} a_{u}\right) \frac{\partial}{\partial a}+\left(b_{t}+u_{t} b_{u}\right) \frac{\partial}{\partial b}+\left(c_{t}+u_{t} c_{u}\right) \frac{\partial}{\partial c}+\cdots, \\
& D_{x}^{e}=\frac{\partial}{\partial x}+u_{x} \frac{\partial}{\partial u}+\left(a_{x}+u_{x} a_{u}\right) \frac{\partial}{\partial a}+\left(b_{x}+u_{x} b_{u}\right) \frac{\partial}{\partial b}+\left(c_{x}+u_{x} c_{u}\right) \frac{\partial}{\partial c}+\cdots
\end{aligned}
$$

The operators $D_{t}, D_{x}$ and $D_{u}$ are operators of total derivatives with respect to $t, x$ and $u$, where the space of the independent variables consists of $t, x$, and $u$,

$$
\begin{aligned}
D_{t} & =\frac{\partial}{\partial t}+a_{t} \frac{\partial}{\partial a}+b_{t} \frac{\partial}{\partial b}+c_{t} \frac{\partial}{\partial c}+\cdots, \\
D_{x} & =\frac{\partial}{\partial x}+a_{x} \frac{\partial}{\partial a}+b_{x} \frac{\partial}{\partial b}+c_{x} \frac{\partial}{\partial c}+\cdots, \\
D_{u} & =\frac{\partial}{\partial u}+a_{u} \frac{\partial}{\partial a}+b_{u} \frac{\partial}{\partial b}+c_{u} \frac{\partial}{\partial c}+\cdots
\end{aligned}
$$

Because of (16) and the definitions of $\zeta^{a_{u}}, \zeta^{b_{u}}, \zeta^{c_{u}}$, one can split the part of determining equations

$$
\zeta^{a_{u}}=0, \quad \zeta^{b_{u}}=0, \quad \zeta^{c_{u}}=0
$$

with respect to $a_{t}, a_{x}, b_{t}, b_{x}, c_{t}, c_{x}$. Consequently the coefficients $\xi^{t}, \xi^{x}, \zeta^{a}, \zeta^{b}$ and $\zeta^{c}$ do not depend on $u$.

Solving the determining equations

$$
\left.\widetilde{X}^{e} F\right|_{(1),(2)}=0,
$$

one finds

$$
\begin{aligned}
& \xi^{t}=p, \quad \xi^{x}=q, \quad \zeta^{u}=u \sigma(t, x), \quad \zeta^{a}=2 a q_{t}-a p_{t}, \quad \zeta^{b}=a q_{x x}+b q_{x}-b p_{t}+q_{t}-2 a \sigma_{x}, \\
& \zeta^{c}=-c p_{t}-\sigma_{t}-a \sigma_{x x}-b \sigma_{x},
\end{aligned}
$$

with arbitrary functions $p=p(t), q=q(t, x), \sigma=\sigma(t, x)$. Hence, we arrive at the following generator of the equivalence group:

$$
X^{e}=p \frac{\partial}{\partial t}+q \frac{\partial}{\partial x}+u \sigma \frac{\partial}{\partial u}+a\left(2 q_{x}-p_{t}\right) \frac{\partial}{\partial a}+\left(a q_{x x}+b q_{x}-b p_{t}+q_{t}-2 a \sigma_{x}\right) \frac{\partial}{\partial b}-\left(c p_{t}+\sigma_{t}+a \sigma_{x x}+b \sigma_{x}\right) \frac{\partial}{\partial c} .
$$

This manuscript is devoted to constructing differential invariants of the equivalence group. For obtaining $n$ thorder invariants we use the infinitesimal test

$$
\widetilde{X}^{e}(J)=0,
$$

where $J$ depends on $a, b, c$ and their derivatives up to order $n$. Notice that for relative invariants the infinitesimal test is

$$
\left.\tilde{X}^{e}\left(J_{k}\right)\right|_{S}=0, \quad k=1, \ldots, s,
$$

where $S$ is a manifold defined by equations $J_{k}=0, k=1, \ldots, s$. 


\subsection{Semi-invariants and the representation of invariants}

Recall that the generator for finding semi-invariants is (see [2])

$$
X^{e}=u \sigma \frac{\partial}{\partial u}-\left(2 a \sigma_{x}\right) \frac{\partial}{\partial b}-\left(\sigma_{t}+a \sigma_{x x}+b \sigma_{x}\right) \frac{\partial}{\partial c},
$$

and that Eq. (1) has the following semi-invariants up to the second-order (see Section 1)

$$
a, \quad a_{t}, \quad a_{x}, \quad a_{t t}, \quad a_{t x}, \quad a_{x x}, \quad K,
$$

where $K$ is given by Eq. (4):

$$
K=2 c_{x} a^{2}-b_{t} a-b_{x x} a^{2}-b_{x} b a+b_{x} a_{x} a+\frac{1}{2} b^{2} a_{x}+b a_{t}+b a_{x x} a-b a_{x}^{2} .
$$

Furthermore, the invariants of the equivalence group defined by the generator (18) are in the class of functions $J$ of the form (4) involving, in general, the derivatives of $a$ up to the order $n$, and derivatives of the function $K(t, x)$ are up to the order $n-2$. Accordingly, the generator (18) is rewritten in the form

$$
X^{e}=a\left(2 q_{x}-p_{t}\right) \frac{\partial}{\partial a}+\zeta^{K} \frac{\partial}{\partial K}
$$

where

$$
\zeta^{K}=q_{t x} a a_{x}-q_{x x x x} a^{3}-q_{x x x} a_{x} a^{2}-2 q_{t x x} a^{2}+3 q_{x} K-q_{t t} a+q_{t}\left(a_{t}+a_{x x} a-a_{x}^{2}\right)-3 p_{t} K .
$$

The coefficients of the prolonged operator

$$
\tilde{X}^{e}=X^{e}+\zeta^{a_{t}} \frac{\partial}{\partial a_{t}}+\zeta^{a_{x}} \frac{\partial}{\partial a_{x}}+\cdots+\zeta^{K_{t}} \frac{\partial}{\partial K_{t}}+\zeta^{K_{x}} \frac{\partial}{\partial K_{x}}+\cdots
$$

are defined by the prolongation formulae, e.g.

$$
\begin{aligned}
& \zeta^{a_{t}}=D_{t} \zeta^{a}-a_{t} D_{t} \xi^{t}-a_{x} D_{t} \xi^{x}, \quad \zeta^{a_{x}}=D_{x} \xi^{a}-a_{t} D_{x} \xi^{t}-a_{x} D_{x} \xi^{x}, \\
& \zeta^{K_{t}}=D_{t} \zeta^{K}-K_{t} D_{t} \xi^{t}-K_{x} D_{t} \xi^{x}, \quad \zeta^{K_{x}}=D_{x} \zeta^{K}-K_{t} D_{x} \xi^{t}-K_{x} D_{x} \xi^{x},
\end{aligned}
$$

where

$$
D_{t}=\frac{\partial}{\partial t}+a_{t} \frac{\partial}{\partial a}+K_{t} \partial_{K}+\cdots, \quad D_{x}=\frac{\partial}{\partial x}+a_{x} \frac{\partial}{\partial a}+K_{x} \partial_{K}+\cdots
$$

For finding invariants one has to apply the following procedure. Let us consider an invariant of order $n$, where it is assumed that $J$ depends on the variable $a$, its derivatives up to $n$ th-order, the function $K$ and its derivatives up to $(n-2)$ order. Invariants can be obtained by solving the equations

$$
\widetilde{X}^{e}(J)=0,
$$

and relative invariants by solving the equations

$$
\left.\tilde{X}^{e}\left(J_{k}\right)\right|_{S}=0 .
$$

\section{Sixth-order invariants}

This section is devoted to finding sixth-order differential invariants. Let

$$
J\left(a, a_{t}, a_{x}, a_{t t}, a_{t x}, a_{x x}, \ldots, a_{x x x x x}, K, K_{t}, K_{x}, K_{t t}, K_{t x}, K_{x x}, \ldots, K_{x x x x}\right)
$$

be a sixth-order differential invariant.

The prolonged operator $\widetilde{X}^{e}$ is defined by (20). Splitting the equations $\widetilde{X}^{e}(J)=0$ with respect to $p, q$ and its derivatives, one obtains a system of 43 linear homogeneous equations. Some of these equations are of the following two types. The first type is

$$
J_{x}+\sum_{i=1}^{n} a_{i} J_{y_{i}}=0,
$$


where $J=J\left(x, y_{1}, y_{2}, \ldots, y_{n}\right)$, and the coefficients $a_{i}(i=1,2, \ldots, n)$ are linear functions of the independent variables $y_{1}, y_{2}, \ldots, y_{i-1}$ which have the form

$$
a_{i}=\sum_{k=1}^{i-1} \beta_{i, k}(x) y_{k}+\gamma_{i}(x) .
$$

The characteristic system for Eq. (21) is

$$
\frac{\mathrm{d} x}{1}=\frac{\mathrm{d} y_{1}}{\gamma_{1}(x)}=\frac{\mathrm{d} y_{2}}{\beta_{2,1}(x) y_{1}+\gamma_{2}(x)}=\frac{\mathrm{d} y_{3}}{\beta_{3,1}(x) y_{1}+\beta_{3,2}(x) y_{2}+\gamma_{3}(x)}=\cdots
$$

From the characteristic system one can obtain the general solution of (21).

The second type of equations is

$$
x J_{x}+\sum_{i=1}^{n} k_{i} y_{i} J_{y_{i}}=0
$$

where $k_{i}(i=1,2, \ldots, n)$ are constant. The general solution of $(22)$ is

$$
J=J\left(J_{1}, J_{2}, \ldots, J_{n}\right), \quad \text { where } J_{i}=\frac{y_{i}}{x^{k_{i}}},(i=1,2, \ldots, n) .
$$

The calculations for obtaining the system of equations for finding invariants and solving its equations are cumbersome. For these calculations we therefore used the Reduce programs developed for solving the linearization problem of third-order ordinary differential equation [5].

After solving the equations of the first and second types the system is reduced to the following system of equations

$$
\frac{\partial J}{\partial J_{2}} J_{1}=0, \quad 6 \frac{\partial J}{\partial J_{3}} J_{3}+5 \frac{\partial J}{\partial J_{1}} J_{1}+7 \frac{\partial J}{\partial J_{2}} J_{2}=0, \quad \frac{\partial J}{\partial J_{2}} J_{3}=0,
$$

where

$$
J_{1}=\lambda /\left(8 a^{5}\right), \quad J_{2}=\left(2 \frac{\partial \lambda}{\partial t} a-5 \frac{\partial a}{\partial t} \lambda\right) /\left(16 a^{7}\right), \quad J_{3}=\left(2 \frac{\partial \lambda}{\partial x} a-5 \frac{\partial a}{\partial x} \lambda\right) /\left(8 a^{6}\right),
$$

and $J=\left(J_{1}, J_{2}, J_{3}\right)$.

If $\lambda=0$, then $J_{1}=J_{2}=J_{3}=0$. This case was studied in [3]. If $\lambda \neq 0$, then $J_{1} \neq 0$. Because of the first equation of (23), $J$ does not depend on $J_{2}$. Solving the second equation of (23), one obtains the only invariant $J_{3}^{5} / J_{1}^{6}$. This invariant was also obtained in [4] as an invariant with respect to contact transformations.

\section{Seventh-order invariants}

Similar to the previous section the system for finding invariants of seventh-order is reduced to the following equations

$$
\begin{aligned}
& 24 \frac{\partial J}{\partial J_{6}} J_{2}+6 \frac{\partial J}{\partial J_{4}} J_{3}+5 \frac{\partial J}{\partial J_{2}} J_{1}=0, \\
& 9 \frac{\partial J}{\partial J_{6}} J_{6}+7 \frac{\partial J}{\partial J_{5}} J_{5}+6 \frac{\partial J}{\partial J_{3}} J_{3}+8 \frac{\partial J}{\partial J_{4}} J_{4}+5 \frac{\partial J}{\partial J_{1}} J_{1}+7 \frac{\partial J}{\partial J_{2}} J_{2}=0, \\
& 3 \frac{\partial J}{\partial J_{6}} J_{4}+2 \frac{\partial J}{\partial J_{4}} J_{5}+\frac{\partial J}{\partial J_{2}} J_{3}=0,
\end{aligned}
$$


where

$$
\begin{aligned}
J_{4}= & \left(-5 \lambda_{t} a_{x} a-4 \lambda_{x} a_{t} a-5 a_{t x} a \lambda+15 a_{t} a_{x} \lambda+2 \lambda_{t x} a^{2}\right) /\left(8 a^{8}\right), \\
J_{5}= & \left(-9 \lambda_{x} a_{x} a-5 a_{x x} a \lambda+15 a_{x}^{2} \lambda+2 \lambda_{x x} a^{2}\right) /\left(8 a^{7}\right), \\
J_{6}= & \left(-40 a_{t x x} a^{2} \lambda-4 a_{x x x} \lambda_{x} a^{3}+30 a_{x x x} a_{x} a^{2} \lambda-40 \lambda_{t} a_{t} a\right. \\
& +\lambda_{x} a\left(-8 a_{t x} a+8 a_{t} a_{x}+6 a_{x x} a_{x} a-3 a_{x}^{3}-8 K\right)+80 a_{t x} a_{x} a \lambda \\
& -40 a_{t t} a \lambda+100 a_{t}^{2} \lambda+40 a_{t} a_{x x} a \lambda-80 a_{t} a_{x}^{2} \lambda-20 a_{x x x x} a^{3} \lambda \\
& \left.+30 a_{x x}^{2} a^{2} \lambda-75 a_{x x} a_{x}^{2} a \lambda+30 a_{x}^{4} \lambda+80 a_{x} K \lambda-40 K_{x} a \lambda+8 \lambda_{t t} a^{2}\right) /\left(32 a^{9}\right) .
\end{aligned}
$$

Taking the Poisson bracket of Eqs. (24) and (26), one obtains the equation

$$
\frac{\partial J}{\partial J_{6}} J_{3}=0
$$

Assuming $\lambda \neq 0$, Eqs. (24) and (25) can be solved. The remaining Eqs. (26) and (27) are reduced to the equations

$$
\begin{aligned}
& 2 \frac{\partial J}{\partial J_{10}}\left(5 J_{8}-3 J_{7}^{2}\right)+15 \frac{\partial J}{\partial J_{9}} J_{10}=0, \\
& \frac{\partial J}{\partial J_{9}} J_{7}=0,
\end{aligned}
$$

where $J=J\left(J_{7}, J_{8}, J_{9}, J_{10}\right)$, and

$$
J_{7}=\frac{J_{3}}{J_{1}^{6 / 5}}, \quad J_{8}=\frac{J_{5}}{J_{1}^{7 / 5}}, \quad J_{9}=\frac{5 J_{1} J_{6}-12 J_{2}^{2}}{5 J_{1}^{14 / 5}}, \quad J_{10}=\frac{5 J_{1} J_{4}-6 J_{2} J_{3}}{5 J_{1}^{13 / 5}} .
$$

Since Eqs. (28) and (29) contain no derivatives with respect to $J_{7}$ and $J_{8}$, the variables $J_{7}$ and $J_{8}$ are invariants.

If $J_{7} \neq 0$, then $J$ does not depend on $J_{9}$, and Eq. (28) becomes

\begin{tabular}{|c|c|c|c|}
\hline Conditions & & & Additional invariant \\
\hline$J_{7} \neq 0$ & $\begin{array}{l}5 J_{8}-3 J_{7}^{2} \neq 0 \\
5 J_{8}-3 J_{7}^{2}=0\end{array}$ & & $\begin{array}{l}\text { No } \\
J_{10}\end{array}$ \\
\hline$J_{7}=0$ & $\begin{array}{l}J_{8} \neq 0 \\
J_{8}=0\end{array}$ & $\begin{array}{l}J_{10} \neq 0 \\
J_{10}=0\end{array}$ & $\begin{array}{l}J_{11} \\
\text { No } \\
J_{9}\end{array}$ \\
\hline
\end{tabular}

$$
2 \frac{\partial J}{\partial J_{10}}\left(5 J_{8}-3 J_{7}^{2}\right)=0 \text {. }
$$

This equation shows that there is the additional invariant $J_{10}$ which is obtained for $\left(5 J_{8}-3 J_{7}^{2}\right)=0$.

If $J_{7}=0$, then one needs only to solve Eq. (28) which becomes

$$
10 \frac{\partial J}{\partial J_{10}} J_{8}+15 \frac{\partial J}{\partial J_{9}} J_{10}=0 .
$$

If $J_{8} \neq 0$, this equation yields the invariant

$$
J_{11}=J_{9}-\frac{3}{4} \frac{J_{10}^{2}}{J_{8}} .
$$

The assumption $J_{8}=0$ leads to the analysis of the equation

$$
J_{10} \frac{\partial J}{\partial J_{9}}=0 .
$$

If $J_{10}=0$ then one only obtains the invariant $J_{9}$. 
Remark. The invariants $J_{7}, J_{8}, J_{10}$ and $J_{11}$ are equal, up to immaterial constant factors, to the invariants (8), (9), (11),..., respectively. Namely:

$$
J_{7}=8^{1 / 5} \Lambda_{1}, \quad J_{8}=8^{2 / 5} \Lambda_{2}, \quad J_{10}=8^{3 / 5} \Lambda_{3}, \quad J_{9}=8^{4 / 5} \Lambda_{4}, \quad J_{11}=8^{4 / 5} \frac{\Lambda_{5}}{4 \Lambda_{2}} .
$$

\section{Conclusion}

This paper is devoted to finding sixth and seventh-order differential invariants of linear second-order parabolic partial differential equation (1) under an action of the equivalence group of point transformations. We found one sixth-order differential invariant $J_{3}^{5} / J_{1}^{6}$. Seventh-order invariants are $J_{7}, J_{8}$. Other functions $J_{9}, J_{10}$ and $J_{11}$ are invariants for particular cases. We have also found invariants of eighth and ninth-order, but the result is too cumbersome, and it is not presented in the paper.

\section{References}

[1] Lie S. On integration of a class of linear parabolic differential equations by means of definite integrals. CRC handbook of lie group analysis of differential equation. CRC Press; 1881 [vol. 2, 1995].

[2] Ibragimov NH. Laplace type invariants for parabolic equations. Nonlinear Dyn 2002;28:125-33.

[3] Johnpillai IK, Mahomed FM. Singular invariant equation for the $(1+1)$ Fokker-Planck equation. J Phys A Math Gen 2001;34:11033-51.

[4] Morozov OI. Contact equivalence problem for linear parabolic equations. Available from: http://arXiv.org/abs/math-ph/0304045, 2003.

[5] Ibragimov NH, Meleshko SV. Linearization of third-order ordinary differential equations by point and contact transformations. J Math Anal Appl 2005;308:266-89. 